\title{
The boundary layer flow induced above the torsional motion of a disk
}

\author{
M. R. Turner \\ Department of Mathematics \\ University of Surrey \\ Guildford, Surrey GU2 7XH \\ United Kingdom \\ Patrick Weidman \\ Department of Mechanical Engineering \\ University of Colorado \\ Boulder, CO 80309-0427
}

\begin{abstract}
The boundary layer flow above a disk in torsional motion with azimuthal velocity proportional to $r^{m}$ is investigated for all values of $m \geq 1$; here $r$ is the radial coordinate measured from the center of the disk. The resulting flow is a fully three-dimensional exact solution to the steady, axisymmetric boundary layer equations in the form of similarity solutions. We compute wall shear stresses in the radial and azimuthal directions as a function of the torsional exponent $m$, as well as the flow induced in the far field. The large $m$ asymptotics of the problem are computed and compared with numerical solutions. The induced radial velocity profiles have a 'wall-jet' structure and it is found that both the radial and azimuthal velocity components become confined close to the surface of the disk as $m$ increases.
\end{abstract}

\section{Introduction}

Studies which involve examining the flow induced by spinning disks and surfaces has been important for many years, not just for engineering relevance (turbine rotors, circular saws, swept wing flows, air cleaning machines, crystal growth processes, etc) but also for its theoretical importance. The original boundary layer solution above a rotating disk by von Kármán (1921) provides an exact similarity solution to the three-dimensional steady Navier-Stokes equations, and this problem has had significant influence on the understanding of laminarturbulent transition in boundary layers. See Saric, Reed and White (2003) and references therein for a review of this topic.

There has also been considerable attention focused on the analysis of spinning elastic disks and membranes, in particular focusing on the natural frequencies of transverse oscillations which arise, and the corresponding mode shapes of these eigenmodes (Lamb and Southwell, 1921; Adams, 1987; Delapierre et al, 2018). One application of spinning membranes is the deployment of ultralight solar structures on satellites as the rotation causes the surface of the membrane to remain flat, increasing the propulsive action for solar sailing amongst other 
applications. Our focus is away from such space applications and is on the viscous fluid flow induced above the spinning disk, from a fundamental perspective. In previous works on spinning elastic disks, the majority of the focus has been mainly on understanding the transverse deformation of the disk as it stretches outward, not on the flow induced above the disk (Lamb and Southwell, 1921; Adams, 1987). There are also relatively few studies which consider laminar stable flows adjacent to spiraling or twisting bodies or disks in general.

Examples of flows which do consider spiraling or twisting bodies include Weidman (2014) who considered an axisymmetric stagnation-point flow on a spiraling disk. In that study both the irrotational Homann (1936) stagnation flow and the rotational Agrawal (1957) stagnation flow were considered and exact similarity solutions to the Navier-Stokes equations were found. More recently Sajid, et al (2018) extended this work by studying Homann stagnation-point flow of a micropolar fluid on a spiraling disk. More akin to the problem investigated here is the work of Sprague and Weidman (2011) who considered the three-dimensional flow induced in the quiescent fluid exterior by the torsional motion of a cylinder whereby the surface azimuthal velocity of the cylinder is $\gamma z$, where $z$ is the axial coordinate along the cylinder and $\gamma$ is the linear twist rate. An interesting feature of this flow is that the axial pressure gradient induces a weak transverse flow in the meridional plane.

The present investigation focuses on the flow induced above a twisting disk rotating about its center. The azimuthal velocity of the disk, $v$, is assumed to have a power-law behavior of the form $v \sim r^{m}$ for $m \geq 1$, and thus different radii on the disk at different azimuthal velocities with the torsional shearing motion increases with increasing exponent $m$. We find a three-dimensional exact solution to the axisymmetric boundary-layer equations in the form of a similarity solution, valid away from the center of the disk. This problem is the axisymmetric torsional equivalent to the power-law radial stretching problem of Banks (1983) wherein the radial stretching takes the form $u \sim r^{m}$, where $u$ is the radial velocity at the surface of the disk, and both similarity and non-similarity results of the resulting equations were presented.

The presentation of the current work is as follows. The problem formulation is given in $\S 2$ wherein it is shown that the radial pressure gradient far from the center of the disk is negligible in accord with the boundary-layer assumption made for this study. In $\S 2.1$ the reduction to the classic von Kármán (1921) flow for a uniformly rotating disk $(m=1)$ is presented. Section 3 gives the large $m$ asymptotic forms for the radial and azimuthal wall shear stresses and the induced far-field motion. The numerical integration procedure is described in $\S 4$ which also introduces the relevant figures for this study. A discussion and concluding remarks are given in $\S 5$.

\section{Formulation}

A schematic diagram of the flow induced above the torsional motion of a disk considered in this paper is given in figure 1. The boundary layer equations for axisymmetric flow in cylindrical polar coordinates $(r, \theta, z)$ with coordinate velocities $(u, v, w)$ are the equation of 
continuity and the three momentum equations

$$
\begin{aligned}
\frac{u}{r}+u_{r}+w_{z} & =0 \\
u u_{r}+w u_{z}-\frac{v^{2}}{r} & =-\frac{1}{\rho} P_{r}+\nu u_{z z} \\
u v_{r}+w v_{z}+\frac{u v}{r} & =\nu v_{z z} \\
u w_{r}+w w_{z} & =-\frac{1}{\rho} P_{z}+\nu w_{z z}
\end{aligned}
$$

where the subscripts denote partial derivatives. Here $\rho$ is the fluid density, $\nu$ its kinematic viscosity and $P$ is the pressure. We seek solutions for an impermeable disk executing pure torsional motion given as

$$
u(r, 0)=0, \quad v(r, 0)=a r^{m}, \quad w(r, 0)=0
$$

so that far above the plate there is no horizontal motion, viz.

$$
u(r, z) \rightarrow 0, \quad v(r, z) \rightarrow 0, \quad(z \rightarrow \infty) .
$$

Following Banks (1983) we posit the boundary-layer similarity solution

$$
u(r, \eta)=a r^{m} f^{\prime}(\eta), \quad v(r, \eta)=a r^{m} g(\eta), \quad \eta=\mu(r) z
$$

with $\mu(r)$ an as yet undetermined function. To satisfy the continuity equation (2.1a) the axial velocity takes the form

$$
w(r, \eta)=a\left(\frac{\dot{\mu} r^{m}}{\mu^{2}}-\frac{(m+1) r^{m-1}}{\mu}\right) f(\eta)-a \frac{\dot{\mu} r^{m}}{\mu^{2}} \eta f^{\prime}(\eta)
$$

where $\dot{\mu}$ denotes differentiation of $\mu$ with respect to $r$.

Inserting the above ansatz into the azimuthal momentum equation (2.1c) gives

$$
2 a^{2} m r^{2 m-1} g f^{\prime}+a^{2}\left[\frac{\dot{\mu}}{\mu} r^{2 m}-(m+1) r^{2 m-1}\right] f g^{\prime}=\nu a \mu^{2} r^{m} g^{\prime \prime}
$$

where a prime denotes differentiation with respect to $\eta$. For similarity, the term in brackets must satisfy

$$
\frac{\dot{\mu}}{\mu} r^{2 m}=K(m+1) r^{2 m-1}
$$

for some constant $K$. Solving for $\mu$ gives

$$
\mu=c r^{K(m+1)}
$$


for a constant $c$. Inserting this into equation (2.6) and rearranging gives

$$
g^{\prime \prime}+\frac{a}{\nu c^{2}}\left[(m+1)(1-K) f g^{\prime}-2 m g f^{\prime}\right] r^{m-1-2 K(m+1)}=0
$$

from which it is clear that the exponent on $r$ must be zero for the viscous terms to balance the inertial terms. This gives

$$
K=\frac{(m-1)}{2(m+1)}
$$

For convenience we now set the coefficient of $f g^{\prime}$ to 2 which fixes the constant $c$, viz.

$$
c=\sqrt{\frac{a(m+3)}{4 \nu}}
$$

thus furnishing the azimuthal momentum equation

$$
g^{\prime \prime}+2 f g^{\prime}-\left(\frac{8 m}{m+3}\right) f^{\prime} g=0
$$

Now that the form of $\mu(r)$ is known, the $z$-momentum equation (2.1d) leads to an equation for the pressure gradient

$$
-\frac{1}{\rho} P_{\eta}=-\frac{a \nu}{m+3} r^{m-1}\left[(m-1) f^{\prime} H-\frac{1}{2}(m+3) H^{\prime \prime}-H H^{\prime}\right]
$$

where

$$
H(\eta)=(m+3) f+(m-1) \eta f^{\prime}
$$

and so

$$
-\frac{P}{\rho}=-\frac{a \nu}{m+3} r^{m-1}\left[(m-1) \int_{\infty}^{\eta} f^{\prime} H \mathrm{~d} \eta-\frac{1}{2}(m+3) H^{\prime}-\frac{1}{2} H^{2}+\frac{1}{2} H^{2}(\infty)\right]+p_{\infty}(r)
$$

where $p_{\infty}(r)$ is the radial form of the pressure at $\eta=\infty$ which we assume is $O\left(r^{m-1}\right)$ to balance the other terms.

Turning now to the radial momentum equation (2.1b) one finds

$$
f^{\prime \prime \prime}+2 f f^{\prime \prime}-\left(\frac{4 m}{m+3}\right) f^{\prime 2}+\left(\frac{4}{m+3}\right) g^{2}+r^{1-2 m} \frac{P_{r}}{\rho \nu a c^{2}}=0 .
$$

However, from (2.14) we note that $P=O\left(r^{m-1}\right)$ and hence the radial pressure gradient term in $(2.15)$ is $O\left(r^{-(m+1)}\right)$. Therefore, by considering a radial position far from the axis of disk rotation $(r \gg 1)$, the flow field exhibits a similarity solution with the radial momentum equation being reduced to the ODE

$$
f^{\prime \prime \prime}+2 f f^{\prime \prime}-\left(\frac{4 m}{m+3}\right) f^{\prime 2}+\left(\frac{4}{m+3}\right) g^{2}=0 .
$$


From velocity conditions (2.2) and (2.3) one finds that equations (2.12) and (2.16) must satisfy the boundary and far-field conditions

$$
f(0)=0, \quad f^{\prime}(0)=0, \quad f^{\prime}(\infty)=0, \quad g(0)=1, \quad g(\infty)=0 .
$$

Using the known values of $K$ and $c$ the far-field induced velocity is found to be

$$
w(r, \infty)=-\sqrt{a \nu(m+3)} r^{(m-1) / 2} f(\infty) .
$$

Of interest in this work are the radial and azimuthal wall shear stresses. The radial stress is given by

$$
\tau_{r z}=\left.\mu \frac{\partial u}{\partial z}\right|_{z=0}=\mu a^{3 / 2} \sqrt{\frac{m+3}{4 \nu}} r^{(3 m-1) / 2} f^{\prime \prime}(0)
$$

and the azimuthal stress is

$$
\tau_{\theta z}=\left.\mu \frac{\partial v}{\partial z}\right|_{z=0}=\mu a^{3 / 2} \sqrt{\frac{m+3}{4 \nu}} r^{(3 m-1) / 2} g^{\prime}(0)
$$

in which $\mu$ is the fluid viscosity. As for the far-field induced velocity in (2.18) we see a switching of radial behaviour as $m$ varies, which for the stresses occurs at $m=1 / 3$.

\subsection{Reduction to von Kármán flow}

It is noted that the case $m=1$ corresponds to von Kármán flow above a uniformly rotating disk. In this case we identify $a=\Omega$ as the rotation rate of the disk and one finds

$$
\begin{aligned}
u(r, \eta)=r \Omega f^{\prime}(\eta), & v(r, \eta) & =r \Omega f(\eta) \\
w(r, \eta)=-2 \sqrt{\nu \Omega} f(\eta), & \eta & =\sqrt{\frac{\Omega}{\nu} z}
\end{aligned}
$$

and the corresponding boundary-value problem is

$$
\begin{array}{cl}
f^{\prime \prime \prime}+2 f f^{\prime \prime}-f^{\prime 2}+g^{2} & =0, \quad f(0)=0, \quad f^{\prime}(0)=0, \quad f^{\prime}(\infty)=0 \\
g^{\prime \prime}+2\left(f g^{\prime}-f^{\prime} g\right) & =0, \quad g(0)=1, \quad g(\infty)=0 .
\end{array}
$$

Integrations of this reported in Benton (1966) give values $f^{\prime \prime}(0)=0.5102, g^{\prime}(0)=-0.6159$ and $f(\infty)=0.44225$. Note that here $w(r, \infty)$ is independent of $r$, while for the general torsional case this now becomes a function of $r^{(m-1) / 2}$. 


\section{$3 \quad$ Large $m$ asymptotic results}

In the case of large torsional motions (large $m$ ), we can generate asymptotic solutions to the governing equations. For this analysis we introduce the new variables $F(\xi), G(\xi)$ such that

$$
f(\eta)=m^{-1 / 4} F(\xi), \quad g(\eta)=G(\xi), \quad \text { with } \quad \eta=m^{1 / 4} \xi,
$$

which when substituted into (2.12) and (2.16) leads to the pair of equations

$$
\begin{gathered}
F_{\xi \xi \xi}+2 F F_{\xi \xi}-4\left(1+3 m^{-1}\right)^{-1} F_{\xi}^{2}+4\left(1+3 m^{-1}\right)^{-1} G^{2}=0 \\
G_{\xi \xi}+2 F G_{\xi}-8\left(1+3 m^{-1}\right)^{-1} F_{\xi} G=0 .
\end{gathered}
$$

Now expand the solution functions as

$$
\begin{aligned}
& F(\xi)=F_{0}(\xi)+m^{-1} F_{1}(\xi)+O\left(m^{-2}\right) \\
& G(\xi)=G_{0}(\xi)+m^{-1} G_{1}(\xi)+O\left(m^{-2}\right)
\end{aligned}
$$

and insert into equation (3.1) which gives at leading order

$$
F_{0 \xi \xi \xi}+2 F_{0} F_{0 \xi \xi}-4 F_{0 \xi}^{2}+4 G_{0}^{2}=0, \quad F_{0}(0)=0, \quad F_{0 \xi}(0)=0, \quad F_{0 \xi}(\infty)=0
$$

and

$$
G_{0 \xi \xi}+2 F_{0} G_{0 \xi}-8 F_{0 \xi} G_{0}=0, \quad G_{0}(0)=1, \quad G_{0}(\infty)=0 .
$$

Then at $O\left(\mathrm{~m}^{-1}\right)$ one obtains

$$
\begin{gathered}
F_{1 \xi \xi \xi}+2\left(F_{0} F_{1 \xi \xi}+F_{0 \xi \xi} F_{1}\right)-8 F_{0 \xi} F_{1 \xi}+8 G_{0} G_{1}+12\left(F_{0 \xi}^{2}-G_{0}^{2}\right)=0 \\
F_{1}(0)=0, \quad F_{1 \xi}(0)=0, \quad F_{1 \xi}(\infty)=0
\end{gathered}
$$

and

$$
\begin{gathered}
G_{1 \xi}+2\left(F_{0} G_{1 \xi}+G_{0 \xi} F_{1}\right)-24 F_{0 \xi} G_{0}-8\left(F_{0 \xi} G_{1}+F_{1 \xi} G_{0}\right)=0 \\
G_{1}(0)=0, \quad G_{1}(\infty)=0 .
\end{gathered}
$$

Equations (3.3) and (3.4) can be solved numerically using the procedure documented in $\S 4$ below and give the following asymptotic results for the disk shear stress parameters

$$
\begin{gathered}
f^{\prime \prime}(0)=1.11863 m^{-3 / 4}-1.78738 m^{-7 / 4}+O\left(m^{-11 / 4}\right) \\
g^{\prime}(0)=-1.17465 m^{-1 / 4}+1.70654 m^{-5 / 4}+O\left(m^{-9 / 4}\right) .
\end{gathered}
$$

The asymptotic behavior for the induced far-field motion which gives the far-field induced velocity from $(2.18)$ is

$$
f(\infty)=0.42899 m^{-1 / 4}+0.15795 m^{-5 / 4}+O\left(m^{-9 / 4}\right) .
$$




\section{Numerical Results}

To numerically integrate (2.12) and (2.16) it is more convenient to eliminate the appearance of $m$ in the $\eta$ similarity variable so that the integration domain does not grow $\propto m^{1 / 2}$ for large $m$. Thus we introduce the variable

$$
\zeta=\sqrt{\frac{a}{4 \nu}} r^{(m-1) / 2} z
$$

which corresponds to a redefined value of $c$ in (2.11), namely

$$
\bar{c}=\sqrt{\frac{1}{m+3}} c=\sqrt{\frac{a}{4 \nu}}
$$

i.e. $\eta=(m+3)^{1 / 2} \zeta$.

Using this new variable, along with rescaling $f(\eta)=(m+3)^{1 / 2} f(\zeta)$, one obtains, in lieu of (2.12) and (2.16), the governing equations

$$
f_{\zeta \zeta \zeta}+2(m+3) f f_{\zeta \zeta}-4 m f_{\zeta}^{2}+4 g^{2}=0
$$

and

$$
g_{\zeta \zeta}+2(m+3) f g_{\zeta}-8 m f_{\zeta} g=0
$$

with boundary conditions as in (2.17).

Equations (4.3) and (4.4) are integrated via a $4^{\text {th }}$ order Runge-Kutta approach from $\zeta=0$ to $\zeta=\zeta_{\max }=20$ with a step size $\Delta \zeta=10^{-4}$. The unknown velocity shear stresses at the plate $f_{\zeta \zeta}(0)$ and $g_{\zeta}(0)$ are then updated via Newton's method using the calculated values $g\left(\zeta_{\max }\right)$ and $f^{\prime}\left(\zeta_{\max }\right)$. The procedure is repeated until updates to the shear stresses between successive iterations is less that $10^{-10}$. In the presentation that follows all results are reported with respect to the original $\eta$ variable, where

$$
f^{\prime \prime}(0)=(m+3)^{-1 / 2} f_{\zeta \zeta}(0) \quad \text { and } \quad g^{\prime}(0)=(m+3)^{-1 / 2} g_{\zeta}(0)
$$

unless otherwise stated.

The radial wall shear stress parameter $f^{\prime \prime}(0)$ and azimuthal wall stress parameter $g^{\prime}(0)$ are given in figures 2 and 3 respectively. The radial wall stress parameter has a maximum value at $m=1$ and then decays toward zero as $m$ increases. The azimuthal wall stress parameter on the other hand has $g^{\prime}(0)=-0.615922$ at $m=1$ but then decreases from this value to a minimum value of $g^{\prime}(0)=-0.641802$ at $m=2.59$ before then increasing toward zero from below as $m$ increases. In each figure the solid dot signifies the von Kármán $(m=1)$ result. While the stress parameters ultimately decrease in magnitude with increasing $m$ (beyond $m=2.59)$, the physical stresses given by (2.19) and (2.20) require more consideration. The pre-factor of $\sqrt{m+3}$, together with the large $m$ asymptotic expansions (3.5) show that while the stress $\tau_{r z}$ does decay to zero with increasing $m$, the stress $\tau_{\theta z} \propto m^{1 / 4}$ for large $m$, showing an increase of this value in this limit. 
Figure 4 shows the far-field induced flow parameter $f(\infty)$ as a function of $m$. This quantity is related to the far-field axial velocity $w(r, \infty)$ via $(2.18)$, and as $f(\infty)>0$ then $w(r, \infty)<0$ and thus corresponds to a 'down-flow' at $z=\infty$. Note that unlike the von Kármán case for the torsional disk the far-field flow is now $r$-dependent, with the down-flow increasing with increasing $r$.

If we now consider the radial, $f^{\prime}(\zeta)$, and azimuthal, $g^{\prime}(\zeta)$, velocity profiles in figures 5 and 6 respectively, then we observe that both are strictly positive, and that the thickness of the boundary layer thins for increasing $m$. Note that we plot these profiles as a function of $\zeta$ to allow for clear presentation on one figure by taking into account the dependence of $\eta$ on $m$. The boundary layer thins $\propto m^{-1 / 4}$ as predicted by the asymptotic results in $\S 3$ $\left(\eta=m^{1 / 4} \xi=m^{1 / 2} \zeta \Longrightarrow \zeta=m^{-1 / 4} \xi\right)$, and this thinning suggests that the flow is likely to become more convectively stable for large $m$ values. It is however, not clear how the absolute instability properties of the flow would be affected, but this is beyond the scope of the current paper. The flow in the radial direction, figure 5, has a 'wall jet' structure which weakens in magnitude as $m$ increases for a fixed $r$ value (Glauert, 1956). This can be seen in figure 7 which depicts the maximum value of $f^{\prime}(\zeta)$ in the $\zeta$ direction, as a function of $m$. As the radial flow at the surface of the disk is directed away from the center then this needs to be accompanied by a corresponding down-flow at $z=\infty$ in order for the total fluid mass to be conserved, which is the case as seen in figure 4 .

A comparison of the numerical results with the large- $m$ asymptotic results are given in $\log -\log$ format for $f^{\prime \prime}(0)$ in figure $8,-g^{\prime}(0)$ in figure 9 , and $f(\infty)$ in figure 10 . In each figure the first order asymptotic result is plotted as the dashed line while the second order asymptotic result is plotted as the dash-dot-dash line. The results here show that the twoterm asymptotic results are in excellent agreement with the full numerical results for $m \gtrsim 50$.

\section{Discussion and Conclusion}

We have investigated laminar flow solutions for the flow induced above a disk whose azimuthal velocity has the form $a^{m}$ for constants $a, m \geq 1$, and exact three-dimensional solutions to the steady, axisymmetric boundary layer equations are found in the form of similarity solutions. Numerical computations are presented up to $m=1000$. The resulting radial shear stress parameter $f^{\prime \prime}(0)$ display a reduction in magnitude as $m$ increases, while the corresponding azimuthal shear stress parameter $g^{\prime}(0)$ decreases from its value at $m=1$ to a minimum value at $m=2.59$ before increasing to zero from below as $m$ increases further. However, the physical stresses at the surface of the disk given by (2.19) and (2.20) are $\tau_{r z} \sim m^{-1 / 4}$ and $\tau_{\theta z} \sim m^{1 / 4}$, and so the azimuthal stress in fact increases in magnitude with increasing $m$, not decreasing as the $g^{\prime}(0)$ parameter suggests. This increase in azimuthal shear stress means the azimuthal velocity profile becomes isolated closer to the surface of the disk (i.e. the boundary-layer thickness decreases) and hence the flow is likely to become more convectively stable in this limit. The actual shape of the velocity profiles are similar to that found in the rigidly rotating disk $(m=1)$ problem, with a radial wall jet structure 
and an azimuthal profile which decays monotonically from its value at the disk. 


\section{References}

Adams, G. G., Critical speeds for a flexible spinning disk, Int. J. Mech. Sci., 29(8), 525-532 (1987).

Agrawal, H. L., A new exact solution of the equations of viscous motion with axial symmetry, Q. J. Mech. Appl. Math., 10, 42-44 (1957).

Banks, W. H. H., Similarity solutions of the boundary-layer equations for a stretching wall, J. Mec. Thor. Appl. 2(3), 375-392 (1983).

Benton, E. R., On the flow due to a rotating disk, J. Fluid Mech., 24, 781-800 (1966).

Delapierre, M., Chakraborty, D., Sader, J. E. and Pellegrino, S., Wrinkling of transversely loaded spinning membranes, Int. J. Solids Struct., 139140 163-173 (2018).

Glauert, M., The wall jet. J. Fluid Mech., 1(6), 625-643 (1956).

Homann, F., Der Einfluss grosser Zahigkeit bei der Stomung um den Zylinder und um de Kugel, Z. Angew. Math., 16, 153-164 (1936).

Kármán, Th. von, Über laminare und turbulente Reibung, Z. Angew Math. Mech., 1, 233-252 (1921).

Lamb, H. and Southwell, R. V., The vibrations of a spinning disk, Proc. R. Soc. Lond., A99, 272-280 (1921).

Sajid, M., Sadiq, M. N., Ali, N. and Javed, T., Numerical simulation for Homann flow of a micropolar fluid on a spiraling disk, Euro. J. Mech. B/Fluids, 72, 320-327 (2018).

Saric, W. S., Reed, H. L. and White, E. B., Stability and transition of three-dimensional boundary layers. Ann. Rev. Fluid Mech. 35, 413-440 (2003).

Sprague, M. A. and Weidman, P. D., Three-dimensional flow induced by the torsional motion of a cylinder, Fluid Dyn. Res., 43, 015501 (2011).

Weidman, P., Axisymmetric stagnation-point flow on a spiraling disk, Phys. Fluids, 26, 073603 (2014). 


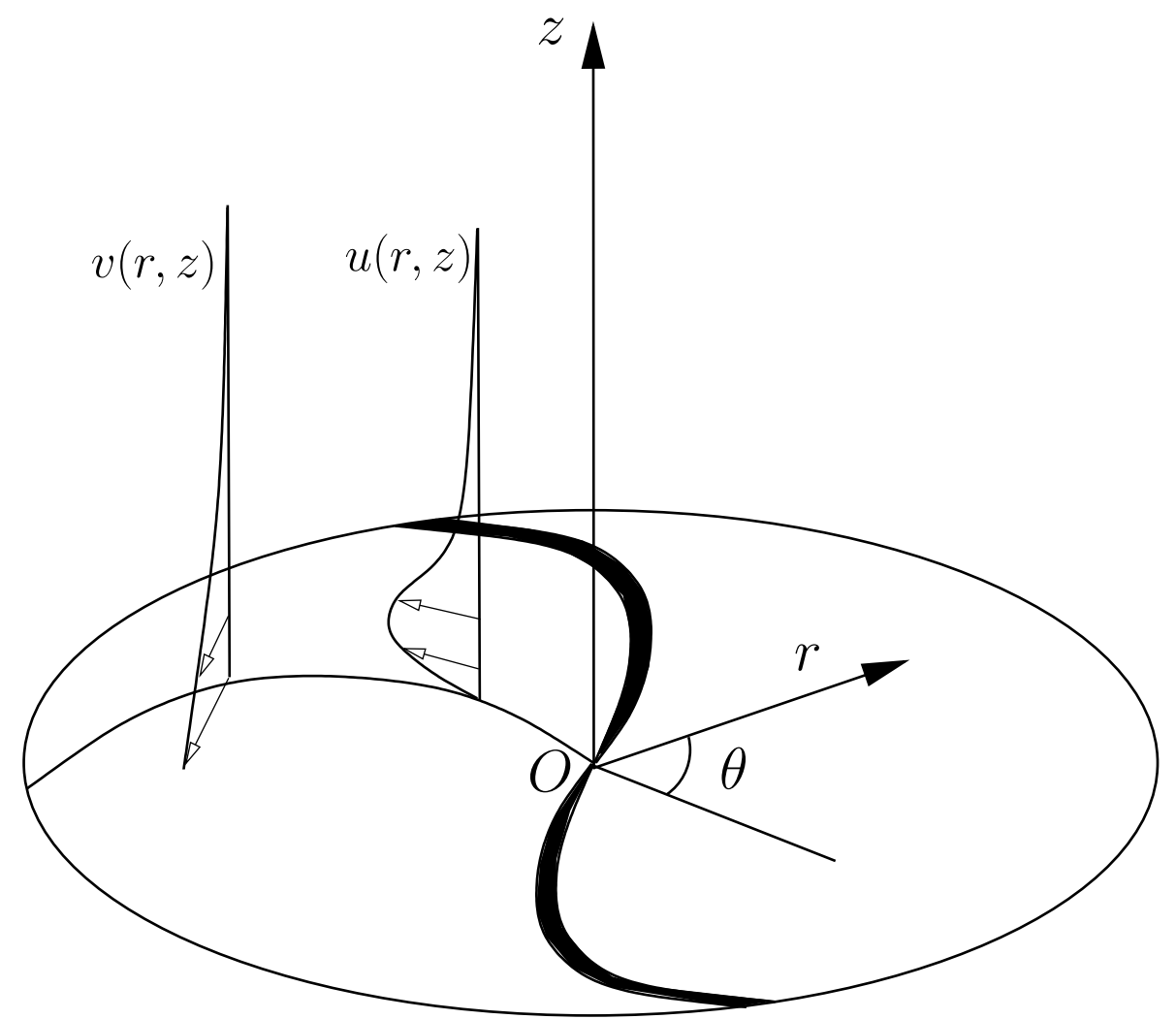

Figure 1. Schematic diagram of the steady, axisymmetric flow induced above the torsional motion of a disk. 


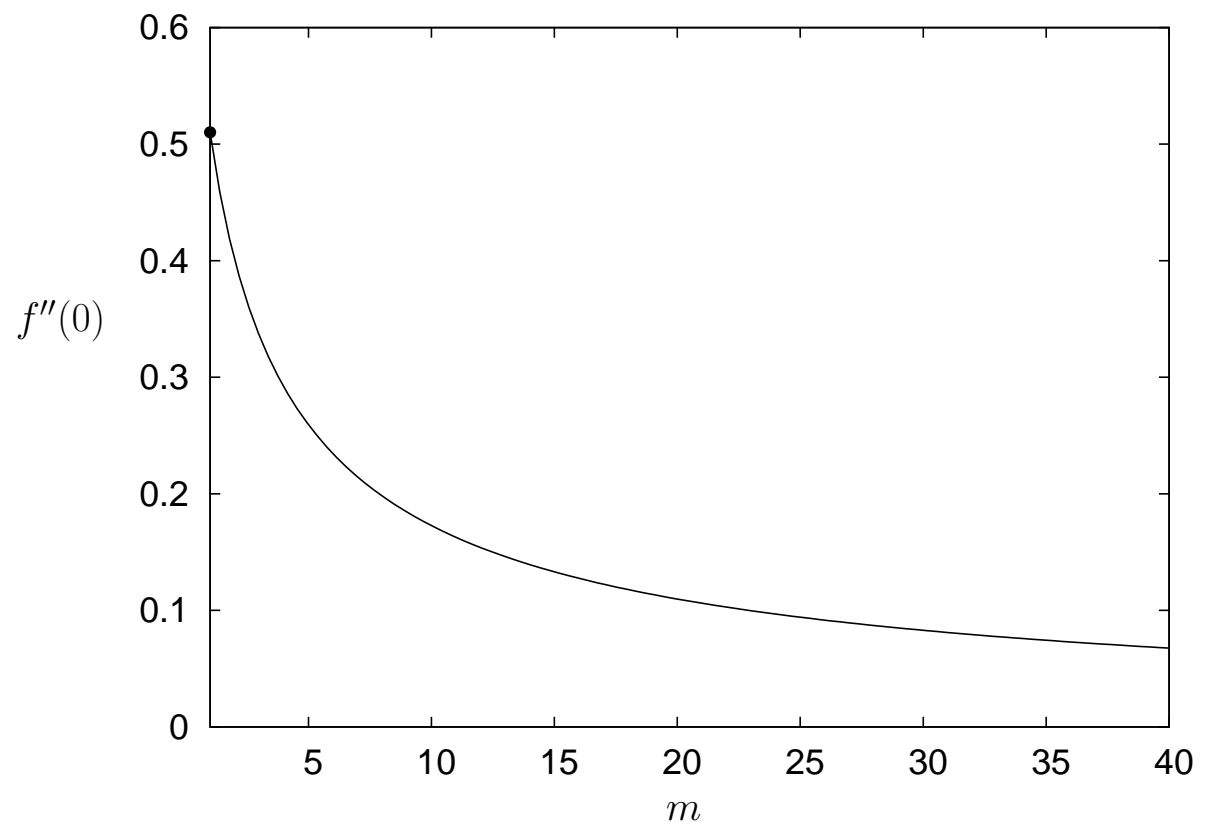

Figure 2. Radial wall shear stress parameter $f^{\prime \prime}(0)$ as a function of torsional exponent $m$. The dot at $m=1$ is for von Kármán flow.

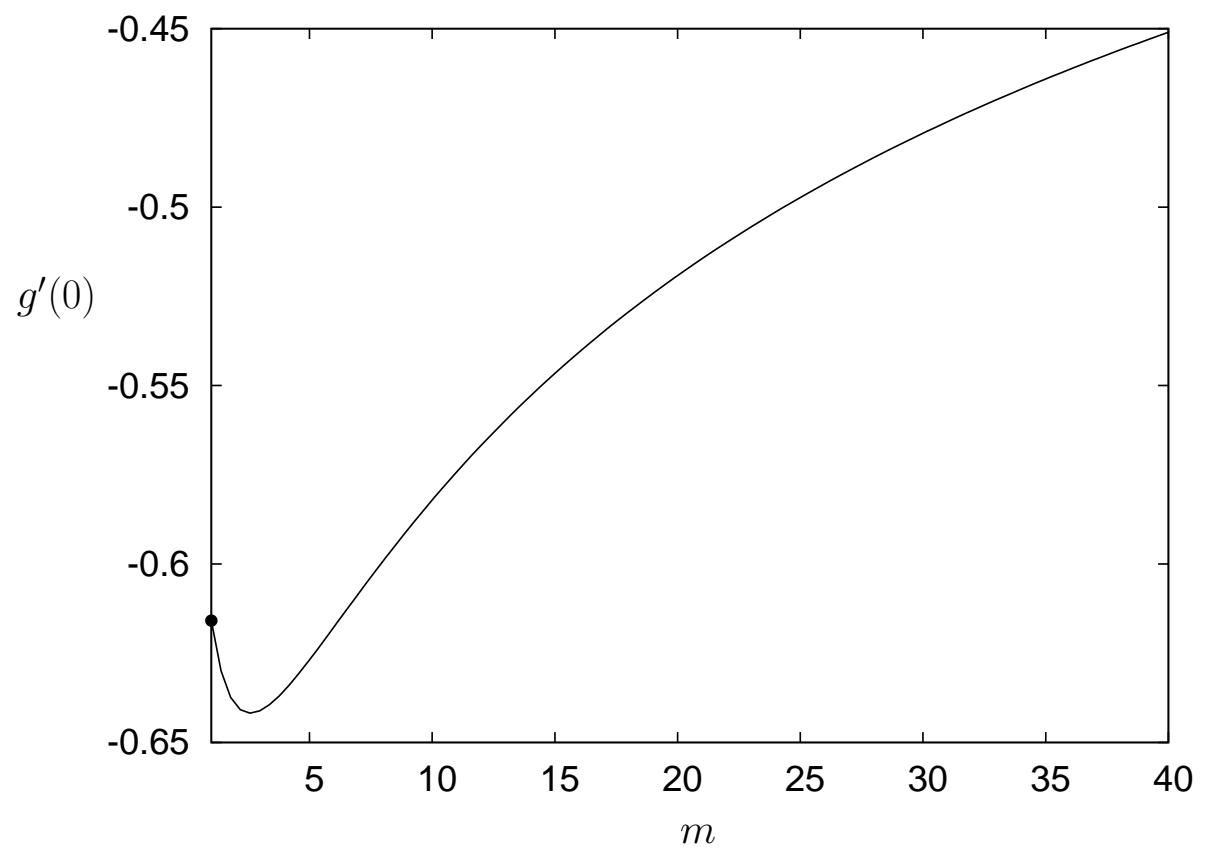

Figure 3. Azimuthal wall shear stress parameter $g^{\prime}(0)$ as a function of torsional exponent $m$. The dot at $m=1$ is for von Kármán flow. 


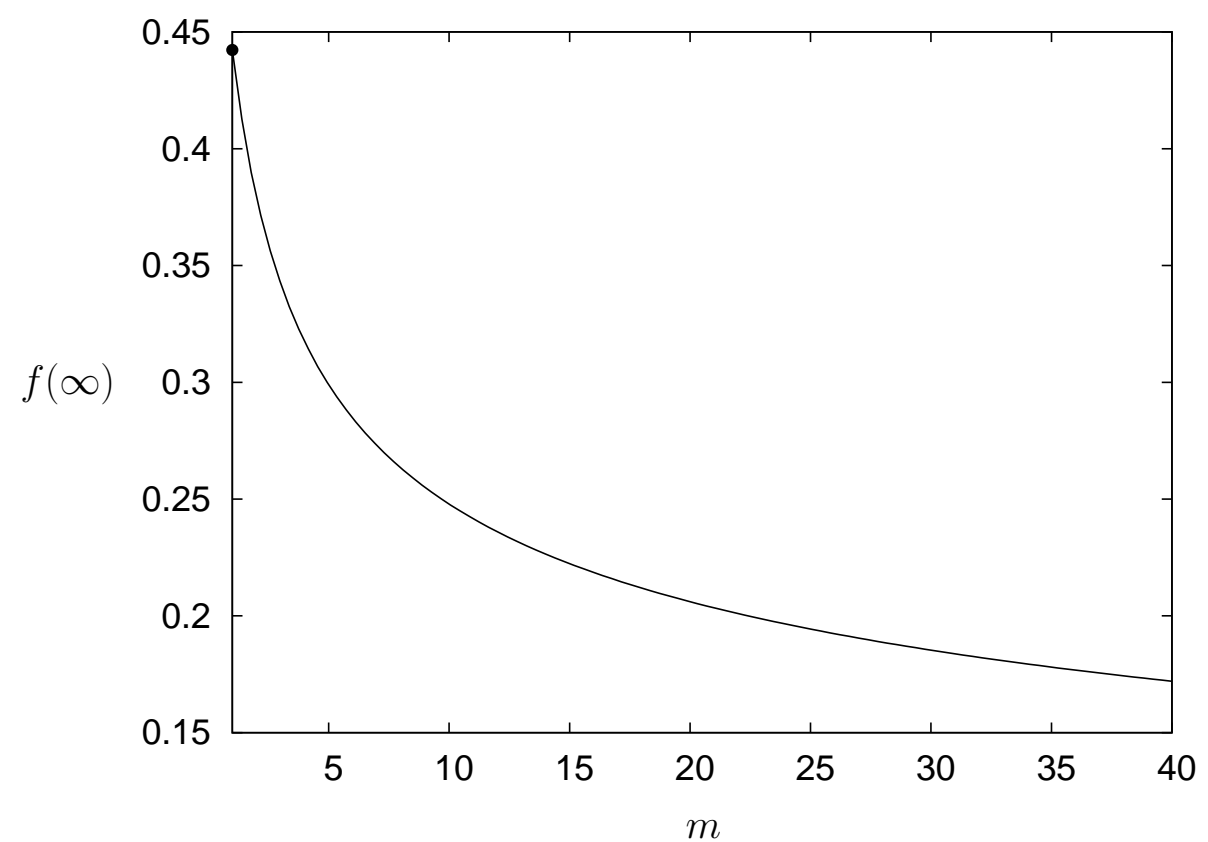

Figure 4. Induced far-field flow parameter $f(\infty)$ as a function of torsional exponent $m$. The dot at $m=1$ is for von Kármán flow.

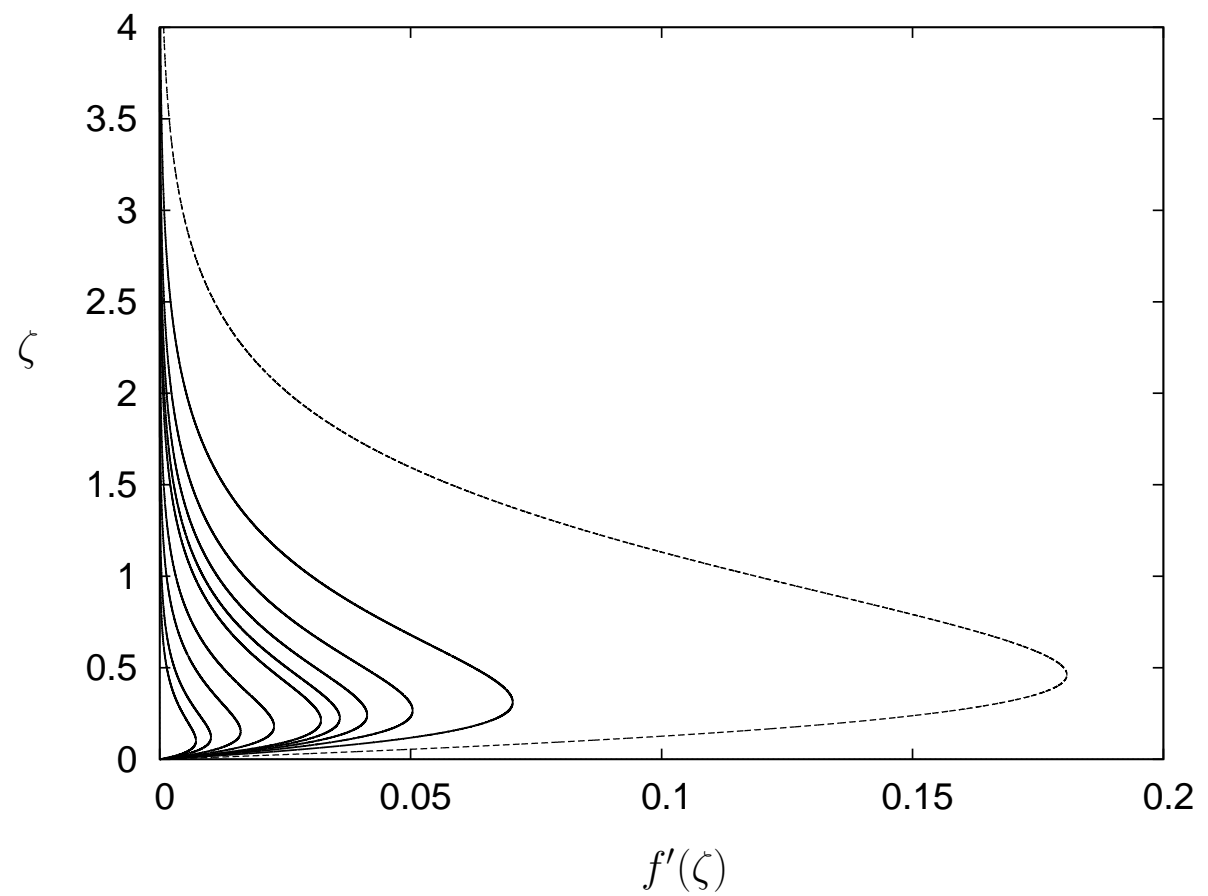

Figure 5 . The $m=\{1,10,20,30,40,50,100,200,500,1000\}$ radial velocity profiles with the $m=1$ von Kármán flow shown as the dashed line. 


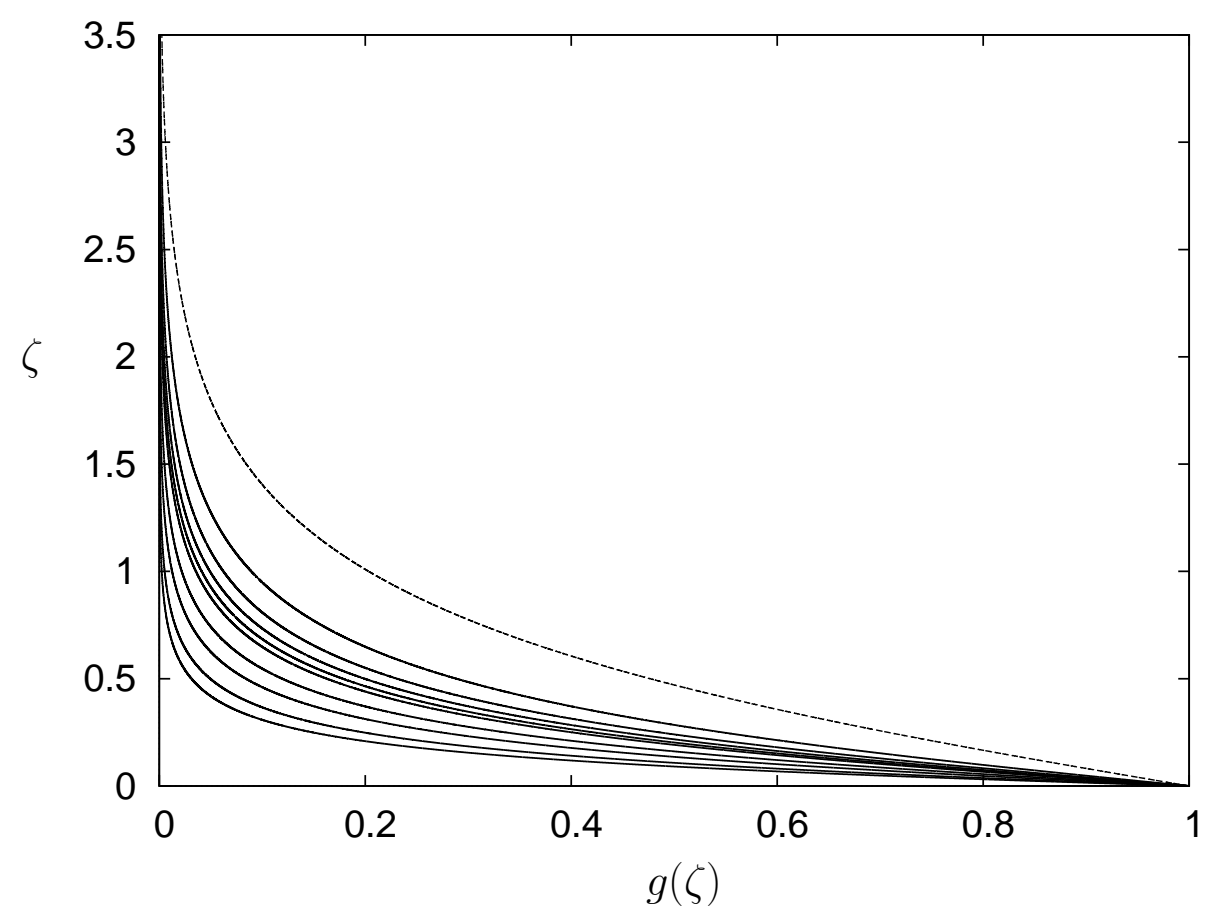

Figure 6 . The $m=\{1,10,20,30,40,50,100,200,500,1000\}$ azimuthal velocity profiles with the $m=1$ von Kármán flow shown as the dashed line.

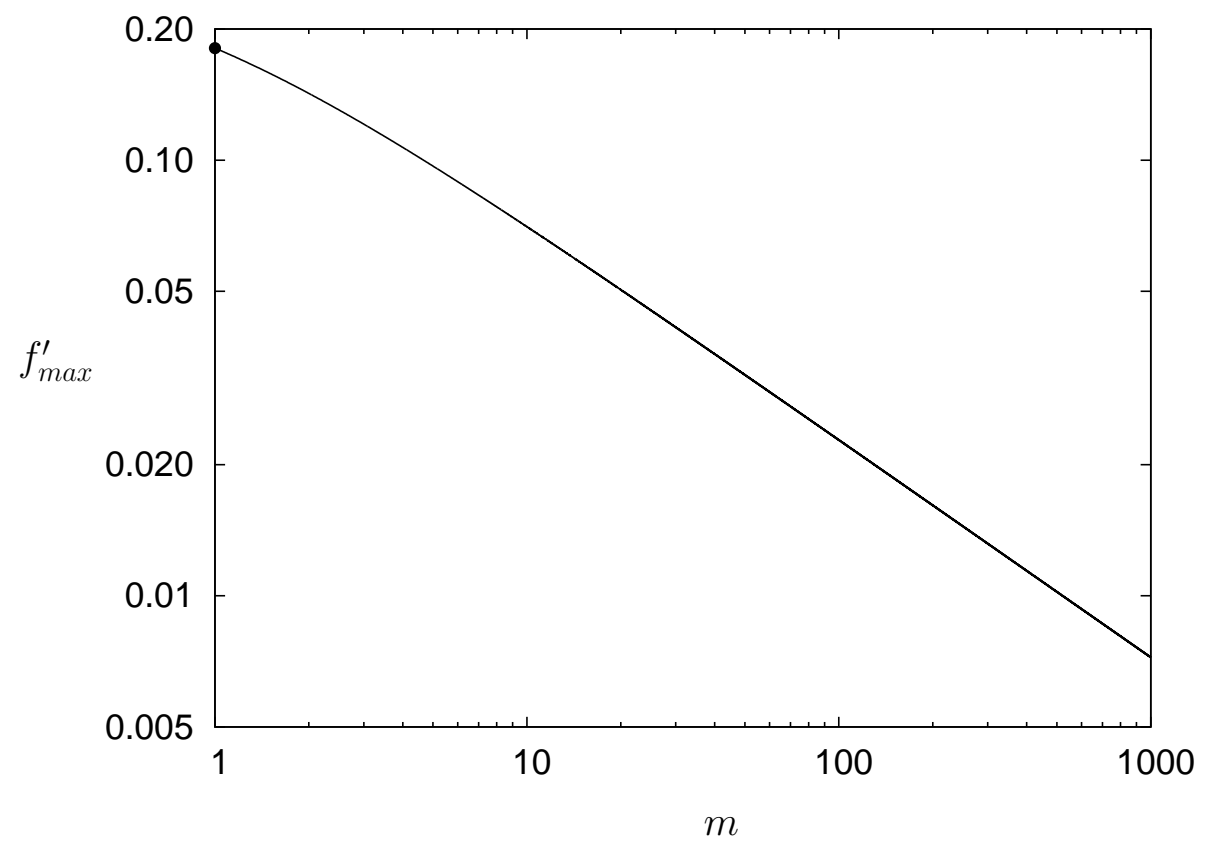

Figure 7. Variation of $f_{\max }^{\prime}$ with $m$ showing the von Kármán result as a dot at $m=1$. 


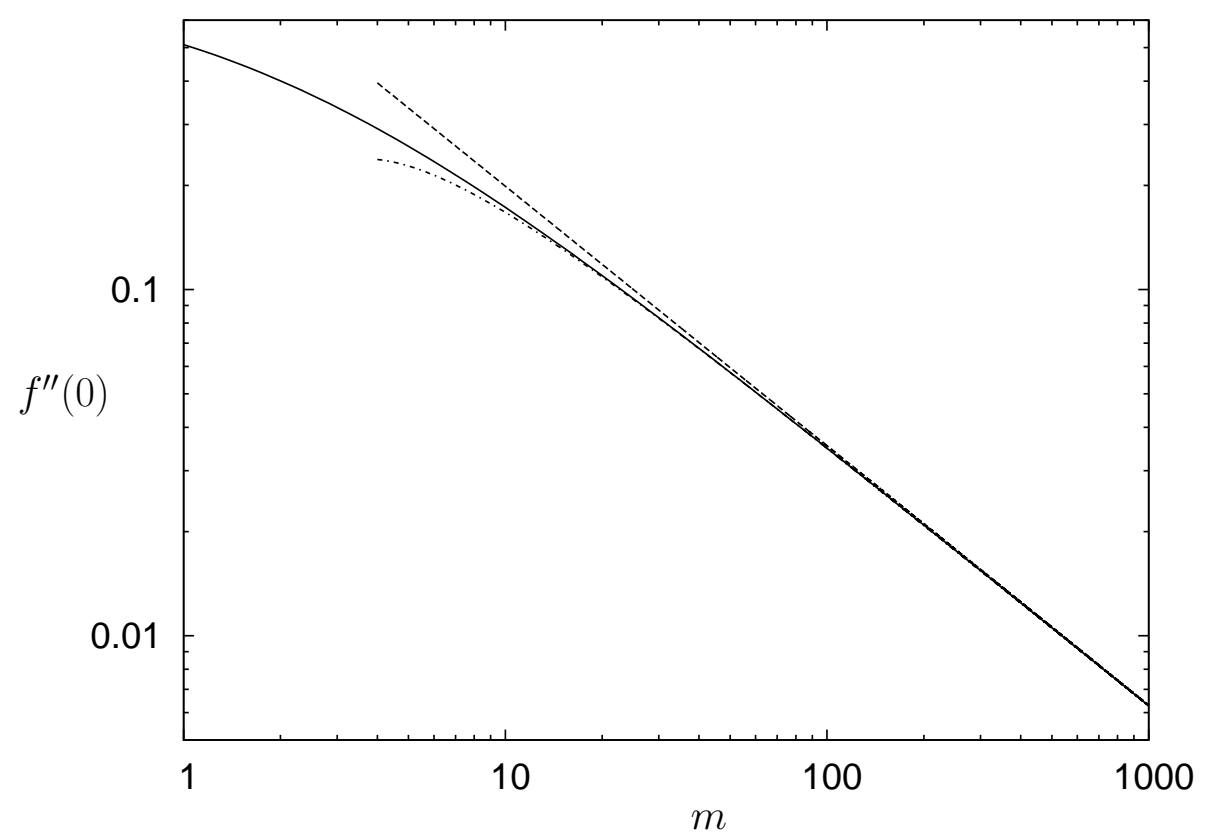

Figure 8. Radial wall shear stress parameter $f^{\prime \prime}(0)$ as a function of torsional exponent $m$. The first order large- $m$ asymptotics are shown by the dashed line while the second order asymptotics are shown by the dash-dot-dash line.

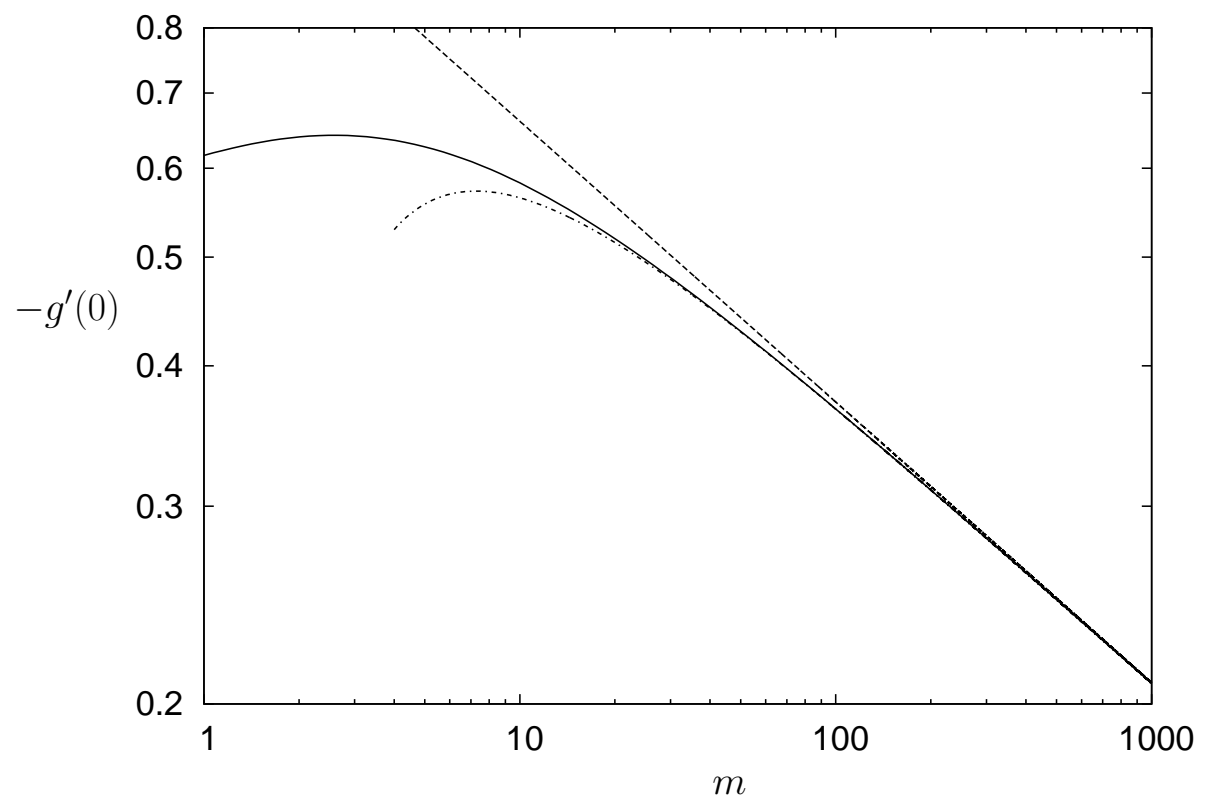

Figure 9. Azimuthal wall shear stress parameter $g^{\prime}(0)$ as a function of torsional exponent $m$. The first order large- $m$ asymptotics are shown by the dashed line while the second order asymptotics are shown by the dash-dot-dash line. 


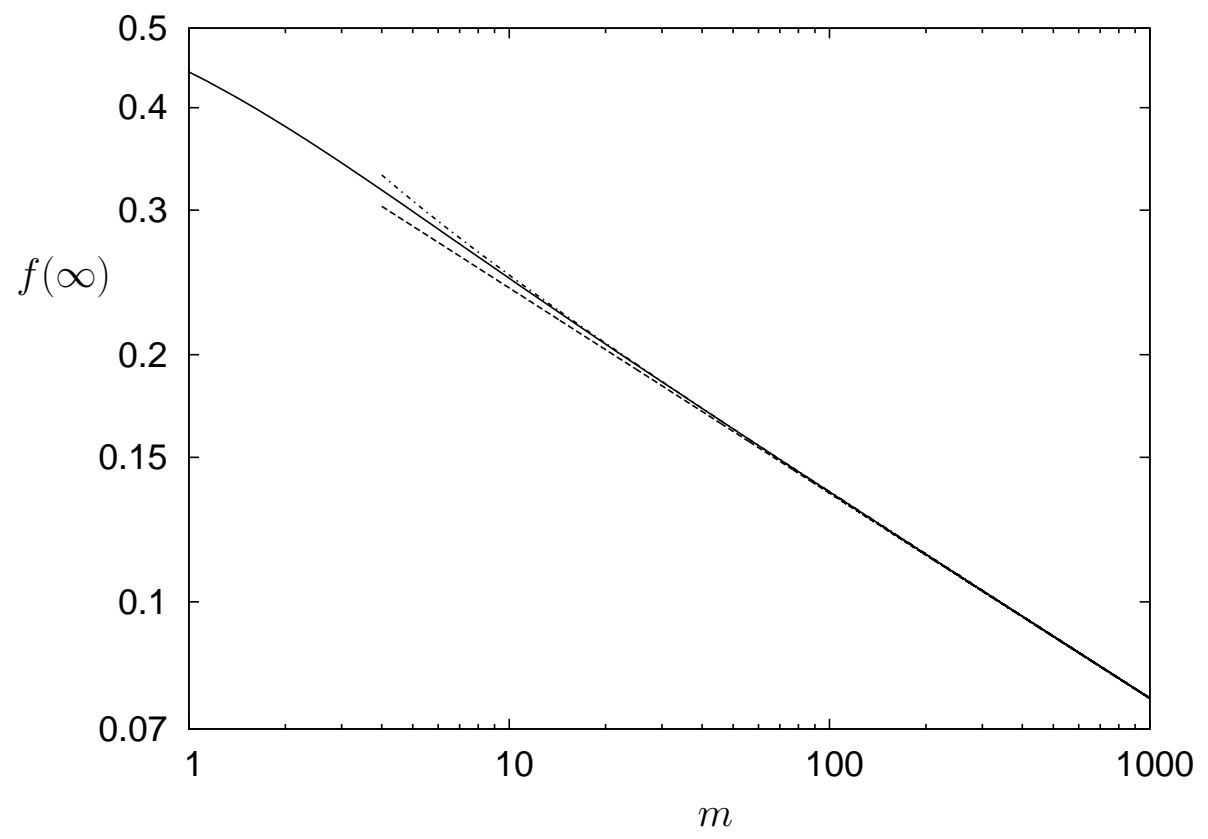

Figure 10. Induced far-field flow parameter $f(\infty)$ as a function of torsional exponent $m$. The first order large- $m$ asymptotics are shown by the dashed line while the second order asymptotics are shown by the dash-dot-dash line. 